\title{
6mA-Pred: Identifying DNA N6-methyladenine sites based on deep learning
}

\author{
Qianfei Huang ${ }^{\text {Equal first author, } 1}$, Wenyang Zhou ${ }^{\text {Equal first author, } 2}$, Fei Guo ${ }^{1}$, Lei Xu ${ }^{\text {Corresp., } 3}$, Lichao Zhang ${ }^{\text {Corresp. } 4}$ \\ ${ }^{1}$ College of Intelligence and Computing, Tianjin University, Tianjin, China, Tianjin, China \\ 2 School of Life Science and Technology, Harbin Institute of Technology, Harbin, China, Harbin, China \\ 3 School of Electronic and Communication Engineering, Shenzhen Polytechnic, Shenzhen, China, Shenzhen, China \\ ${ }^{4}$ School of Intelligent Manufacturing and Equipment, Shenzhen Institute of Information Technology, Shenzhen 518172, China, Shenzhen, China \\ Corresponding Authors: Lei Xu, Lichao Zhang \\ Email address: csleixu@szpt.edu.cn, Iczhang5354@szu.edu.cn
}

With the accumulation of data on $6 \mathrm{~mA}$ modification sites, an increasing number of scholars have begun to focus on the identification of $6 \mathrm{~mA}$ sites. Despite the recognized importance of $6 \mathrm{~mA}$ sites, methods for their identification remain lacking, with most existing methods being aimed at their identification in individual species. In the present study, we aimed to develop an identification method suitable for multiple species.

Based on previous research, we propose a method for $6 \mathrm{~mA}$ site recognition. Our experiments prove that the proposed $6 \mathrm{~mA}$-Pred method is effective for identifying $6 \mathrm{~mA}$ sites in genes from taxa such as rice, Mus musculus, and human. A series of experimental results show that $6 \mathrm{~mA}$-Pred is an excellent method. We provide the source code used in the study, which can be obtained from

http://39.100.246.211:5004/6mA Pred/ 


\section{6mA-Pred: Identifying DNA N6-Methyladenine Sites 2 Based on Deep Learning}

3 Qianfei Huang ${ }^{1, \#}$, Wenyang Zhou ${ }^{2, \#}$, Fei Guo ${ }^{1}$, Lei Xu ${ }^{3, *}$, Lichao Zhang,,*

4 1. College of Intelligence and Computing, Tianjin University, Tianjin, China

52 School of Life Science and Technology, Harbin Institute of Technology, Harbin,

6 China

73 School of Electronic and Communication Engineering, Shenzhen Polytechnic,

8 Shenzhen, China

94 School of Intelligent Manufacturing and Equipment, Shenzhen Institute of

10 Information Technology, Shenzhen 518172, China

11 *corresponding author: csleixu@szpt.edu.cn, lczhang5354@szu.edu.cn

12 \#equally contributed

\section{Abstract}

With the accumulation of data on $6 \mathrm{~mA}$ modification sites, an increasing number of scholars have begun to focus on the identification of $6 \mathrm{~mA}$ sites. Despite

17 the recognized importance of $6 \mathrm{~mA}$ sites, methods for their identification remain 
18 lacking, with most existing methods being aimed at their identification in

19 individual species. In the present study, we aimed to develop an identification

20 method suitable for multiple species.

21 Based on previous research, we propose a method for $6 \mathrm{~mA}$ site recognition.

22 Our experiments prove that the proposed 6mA-Pred method is effective for 23 identifying $6 \mathrm{~mA}$ sites in genes from taxa such as rice, Mus musculus, and human.

24 A series of experimental results show that $6 \mathrm{~mA}-\mathrm{Pred}$ is an excellent method. To

25 facilitate people to use the model proposed in this experiment, we have established

26 a Web server, the website is http://39.100.246.211:5004/6mA Pred/. This web

27 server contains experimental code and data and can make simple predictions.

Keyword:6mA, Deep Learning, LSTM.

30 Introduction

DNA modification sites play vital roles in multiple biological processes and are attracting increasing research attention. Methylation continues to be a hot topic in epigenetics, and 5mC methylation has been extensively studied(Liu et al. 2019b). With the advancement of sequencing technology, 6mA methylation has slowly 35 attracted increasing attention. 6mA methylation not only affects gene expression 
36 but also regulates development in plants and animals(Xu et al. 2020a). Many

37 diseases, including cancer, are related to $6 \mathrm{~mA}$ methylation(Chen et al. 2019a; Chen

38 et al. 2019b; Xu et al. 2019a). With the progress of $6 \mathrm{~mA}$ methylation-related

39 research, large amounts of data have been collected. However, effective methods

40 for $6 \mathrm{~mA}$ site identification are lacking. bioinformatics. Many methods have been studied and have achieved good results. Although research on $4 \mathrm{mC}(\mathrm{He}$ et al. 2019) and $5 \mathrm{mC}$ is mature, research on the identification of $6 \mathrm{~mA}$ modification sites has just begun. The computational method i6mA-Pred was used to identify $6 \mathrm{~mA}$ modification sites in the rice genome with

47 high accuracy. Several methods for identifying $6 \mathrm{~mA}$ loci in the rice genome have 48 been proposed, such as MM-6mAPred, iDNA-6mA-rice (Hao et al. 2019), 49 SDM6A (Basith et al. 2019), i6mA-DNCP (Kong \& Zhang 2019) and SNNRice6mA (Yu \& Dai 2019). In addition, methods for the identification of

$516 \mathrm{~mA}$ sites in Mus musculus and humans have gradually emerged, such as iDNA6mA-PseKNC (Feng et al. 2019), csDMA (Liu et al. 2019c), SICD6mA , and 6mA-Finder (Xu et al. 2020b). Several datasets are publicly available, and many desirable features and models have been proposed. Application of the feature algorithms NCP and one-hot, feature fusion and deep learning methods has greatly 
56 accelerated the identification of 6mA-modified sites. Among the employed

57 algorithms, SVM and RF exhibit stable performance and perform well on some

58 datasets (Liu et al. 2019a; Shen et al. 2019b; Sun et al. 2020; Wang et al. 2020a;

59 Wang et al. 2020b; Yan et al. 2020; Zhou et al. 2018; Zhou et al. 2017). In addition,

60 the Markov model has achieved excellent results in predicting $6 \mathrm{~mA}$ sites in the rice

61 genome. In the application of feature methods, most researchers use multiple

62 feature fusion methods and analyze various features. In general, the different

63 methods have achieved good results and provided direction for subsequent

64 research.

65 In the research mentioned above, most methods have employed machine 66 learning (Patil \& Chouhan 2019; Zou 2019; Zou \& Ma 2019) and detailed analysis

67 of different feature methods. There are some good models that use deep learning 68 methods, such as SNNRice6mA and SICD6mA. SNNRice6mA employs CNN

69 (Ren et al. 2019) to build a network that works well. SICD6mA uses GRU to 70 achieve a good network structure and has been applied extensively to datasets of 71 two species. In this paper, through a summary of the previous research work, we 72 found that LSTM+Attention can identify the modification sites very well, and a 73 large number of experimental results suggest that this is a very good method. 


\section{MATERIALS AND METHODS}

75

76

77

78

79

80

81

82

83

84

85

86

87

88

89

90

91

92

\section{Datasets}

Much research has aimed to identify $6 \mathrm{~mA}$ sites in rice. In reviewing research from the past two years, we found that the amount of data on $6 \mathrm{~mA}$ sites is increasing. We obtained datasets for three species. The first dataset is a rice dataset obtained from 6mA-RicePred(Huang et al. 2020b). This dataset was first used in i6mA-Pred(Chen et al. 2019c) and was provided by the author(Hu et al. 2019). The second dataset is a Mus musculus dataset obtained from iDNA-PseKNC, and it has achieved good results with this dataset. The third dataset is a human dataset obtained from SICD6mA and is the largest of the three datasets. Table 1 provides a summary of each dataset. The lengths of their sequences are all the same: $41 \mathrm{bp}$. Details of these datasets are provided in their source papers. We have organized the datasets, which can be obtained from https://github.com/huangqianfei0916/6ma-rice.

All three data sets use CD-HIT to remove redundancy. sequences with the similarity above $80 \%$ were excluded by using the CD-HIT program. all negative samples were $41 \mathrm{bp}$ in length and the center was A, but not being detected by the SMRT sequencing technology as of $6 \mathrm{~mA}$. Moreover the rice dataset collected negative samples based on the ratio of GAGG, AGG and AG motifs in the positive 
93 samples. the mouse dataset removed positive samples with modQV greater than

9430.

95 Feature encoding and classification algorithms

96

97

98

99

100

101

102

103

104

105

106

107

108

109

110

111

112

One-hot encoding has been used by many researchers for sequence processing with good results (Cheng 2019b; Cheng et al. 2018a; Li et al. 2020; Liu \& Li 2019; Zou et al. 2019). One-hot encoding encodes each nucleotide separately. A disadvantage of one-hot is the lack of timing. Therefore, we used Kmer word segmentation instead of one-hot to capture the relationship between bases(Zuo et al. 2017). The role of Kmer was to help Embedding generate better word vectors. We investigated both normal word segmentation and Kmer word segmentation, and the experimental results showed that Kmer word segmentation achieved superior performance. Fig. 1 shows the process of Kmer word segmentation. Our test for the selection of the $\mathrm{k}$ value revealed three to be the most suitable value. the experimental results are shown in Fig. 2. When $\mathrm{k}$ is 3 , the dictionary size is 64; this is not a large parameter. In the feature extraction stage, the embedding layer is used to extract features. we chose the init method for our experiment. The effect of using init or fine-tune is almost the same, and in some cases, the init method is superior. If there is an excellent pretrained model, it is also a good choice. The quality of the features largely determines the effect of the model. Embedding is a very important module in deep learning, and word2vec is one of the best 
113 embedding methods. The encoding of features can be learned dynamically, and a 114 method of secondary learning called finetune can be achieved in deep learning. In 115 this paper, we use simple Init embedding and Kmer word segmentation.

116 Most methods currently employed for $6 \mathrm{~mA}$ site recognition are machine 117 learning methods, and most of them are only effective for a single species (Cheng 118 2019a; Cheng et al. 2019). In reviewing the latest research, we found that there are 119 many similarities between the attention mechanism and the recognition of $6 \mathrm{~mA}$ 120 sites. Furthermore, LSTM has achieved excellent performance in dealing with 121 sequence problems(Huang et al. 2020a). In constructing the model, we did not 122 adopt a particularly complex structure, and the complexity and effect of the model 123 are not directly related. After feature extraction with the embedding layer, 124 bidirectional LSTM is used to process the sequence features (Xia et al. 2019). The 125 sequence information obtained after LSTM processing can be used to obtain a 126 good feature vector, and this feature is a representation of the overall sequence 127 information. Each time step of LSTM has an output that represents the sequence 128 information up to the current time. The LSTM algorithm can be formulated as 129 follows:

130 131

$$
\begin{aligned}
& i_{t}=\sigma\left(W_{i i} x_{t}+b_{i i}+W_{h i} h_{t-1}+b_{h i}\right) \\
& f_{t}=\sigma\left(W_{i f} x_{t}+b_{i f}+W_{h f} h_{t-1}+b_{h f}\right)
\end{aligned}
$$


132

$$
g_{t}=\tanh \left(W_{i g} x_{t}+b_{i g}+W_{h g} h_{t-1}+b_{h g}\right)
$$

$$
o_{t}=\sigma\left(W_{i o} x_{t}+b_{i o}+W_{h o} h_{t-1}+b_{h o}\right)
$$

$$
c_{t}=f_{t} * c_{t-1}+i_{t} * g_{t}
$$

135

$$
h_{t}=o_{t} * \tanh \left(c_{t}\right)
$$

140 information or take the last one and then apply the fully connected layer to obtain

141 the result. Many scholars have added other layers after LSTM to obtain good

142 features. However, the design of these levels of network structure varies according 143 to the specific application scenarios and problems. 6mA-Pred applies the attention 144 mechanism to the output of LSTM and connects the fully connected layer after the 145 attention layer. analyzed with attention. The inner output of the final output of LSTM and the results of the previous time step can be used to generate the corresponding 
150 attention score. then, the Softmax layer is added to the attention layer to obtain the 151 weight. The output of LSTM and this weight are weighted to obtain the final 152 context vector. The last layer of the network is the fully connected layer, and this 153 layer can obtain the probability of each category. Fig. 1 shows the structure of the 154 entire network and describes the Kmer word segmentation and attention 155 mechanism. The attention mechanism adopted by $6 \mathrm{~mA}$-Pred is not complicated 156 and acts directly on the output of LSTM. The purpose of 6mA-Pred is to obtain the 157 final feature through the difference between global information and local 158 information. We know that the feature corresponding to the sequence containing 159 the modification site is very different from the feature corresponding to the 160 sequence not containing the modification site. Because of the differences, their 161 final context vectors differ. We used the inner product method to obtain the 162 attention score to reflect the intersection of global information and local 163 information. The inner product is not the only option; other operations are possible.

164 Self-attention in Transformer is also a good choice, but the network structure of the 165 model is more complicated. Dot product can get the intersection between different 166 sequences. 6mA-Pred uses this structure to increase the amount of local 167 information in the final feature. 


\section{Performance Evaluation}

A good model evaluation standard is crucial for assessing the utility of a model. Different indicators can be used to reveal the advantages and disadvantages of a model from different perspectives. Sensitivity (Sn), specificity (Sp), accuracy (Acc), and Mathew's correlation coefficient (MCC) are used to evaluate models in machine learning (Chu et al. 2019; Deng et al. 2020; Gong et al. 2019; Jin et al. 2019; Shan et al. 2019; Su et al. 2019a; Su et al. 2019b; Wei et al. 2018a; Wei et al. 2018b; Xu et al. 2018a; Xu et al. 2018b; Xu et al. 2018c; Zhang et al. 2019a;

Zhang et al. 2019b). These metrics are formulated as follows :

$177 S \mathrm{n}=\frac{T P}{T P+F N}$

$178 \mathrm{Sp}=\frac{T N}{T N+F P}(8)$

$179 \quad \mathrm{Acc}=\frac{T P+T N}{T P+T N+F P+F N}$

$\mathrm{MCC}=\frac{T P * T N-F P * F N}{\sqrt{(T P+F P) *(T P+F N) *(T N+F P) *(T N+F N)}}(10)$ false negative, respectively. Sn, Sp, Acc, and MCC can be calculated from these indicators. In addition, AUC (area under the ROC curve) was used to evaluate our 
185 Shen et al. 2019a). For further experiments, Table 2 records the hyperparameters of 186 the model.

\section{Performance Comparison with Different Datasets}

Methods for identifying sites in the rice genome include iDNA6mA-Rice and

190

SNNRice6mA, which are excellent models. After comparing different features in

feature extraction, the developers of iDNA6mA-Rice chose binary encoding, and

they chose RF (random forest) for the classifier. Both the choice of feature method and the performance of the classifier are excellent. iDNA6mA-Rice was applied to various scale segmentation experiments on a rice dataset and achieved very good results. 6mA-Pred was applied in a similar experiment with the rice dataset. the results are shown in Fig 3. The performance of $6 \mathrm{~mA}-$ Pred was better than iDNA6mA-Rice at all ratios. However, iDNA6mA-Rice is also a very good model, and the performance difference between the two models was very small. SNNRice6mA also performs very well for rice genes. Unlike iDNA6mA-Rice, SNNRice6mA uses a deep learning model. SNNRice6mA uses one-hot in the feature encoding stage and has achieved good results. Regarding the overall network structure, SNNRice6mA uses a stack structure of CNN (convolutional neural networks). The network structure of SNNRice6mA was adjusted to derive 
204 SNNRice6mA-large, which also achieved good results. SNNRice6mA and 205 SNNRice6mA-large were employed for five-fold cross-validation on the rice 206 dataset. Table 3 shows the results of comparisons among the different models. The 207 performance of 6mA-Pred was excellent compared to that of the other models.

The model also performed well on the Mus musculus dataset. iDNA6mA209 PseKNC has achieved good results in predicting 6mA loci in the Mus musculus genome and uses machine learning methods for analysis. iDNA6mA-PseKNC uses NCP as the feature algorithm, and many experiments have been conducted for this 212 feature. In addition, iDNA6mA-PseKNC employs the SVM classifier and achieved 213 very good results. $6 \mathrm{~mA}$-Pred is also effective in identifying $6 \mathrm{~mA}$ sites in the $M u s$ 214 musculus genome. In this study, two experiments were conducted with 6mA-Pred, 215 one involving five-fold cross-validation on the dataset, and one involving 216 independent testing by splitting the dataset. Table 4 shows the results of these two 217 experiments and the results for iDNA6mA-PseKNC. iDNA6mA-PseKNC was 218 evaluated via the jackknife test; for deep learning methods, leave-one-out cross219 validation is time consuming and not representative. For evaluation of $6 \mathrm{~mA}-\mathrm{Pred}$, 220 five-fold cross-validation (Fang et al. 2019; He et al. 2018a; Liu 2019; Xiong et al. 221 2018; Xu et al. 2019b; Zhu et al. 2019) and segmentation of the dataset were 222 employed. As shown in Table 4, the performance of 6mA-Pred remained good. 
Among the models used for identifying the $6 \mathrm{~mA}$ sites of human genes,

SICD6mA is currently the best model. SICD6mA is a deep learning model and uses GRU as the basic unit. SICD6mA performs well not only for human genes but 226 also for rice genes. The developers of SICD6mA contributed data and performed 227 extensive data processing. We used the training set and test set provided by SICD6mA's developers for our experiments. SICD6mA does not use one-hot for encoding; rather, it uses 3-mer. Two basic units, BGRU and UGRU, are used in the 230 network model structure, and a two-layer fully connected layer and a Softmax 231 layer are used to improve the network. The experimental results revealed that the 232 performance of SICD6mA was very good. Table 5 shows the experimental results 233 for $6 \mathrm{~mA}$-Pred, which were very similar to the SICD6mA results. These findings 234 proved that $6 \mathrm{~mA}$-Pred is very effective in identifying $6 \mathrm{~mA}$ sites in human genes.

According to the previous conclusions, we conducted related experiments on 236 traditional machine learning methods. NCP and KMER were used in experiments 237 as excellent feature extraction methods. SVM, RF and XGB were excellent algorithms and performed well in previous studies. Therefore, we use them to carry out further experiments. the experimental results are shown in Fig. 4.

\section{Conclusion}


242 Through the analysis of current studies and the performance of a large number 243 of experimental comparisons, we found that $6 \mathrm{~mA}$-Pred is an effective method for 244 identifying 6mA sites. LSTM performs well in processing sequence features and 245 can obtain good features. In addition, the attention mechanism we used is effective 246 for identifying $6 \mathrm{~mA}$ sites. The combination of LSTM and Attention mechanism 247 can produce a theoretically excellent model, and the experiment proves that this 248 conclusion is correct. Related methods will be considered for RNA and protein 249 modification prediction (Dou et al. 2020; He et al. 2018b; Huang \& Li 2018) in the 250 future.

251 The previous studies on this topic are excellent and provide theoretical and 252 experimental support for our research. The attention mechanism in 6mA-Pred can 253 be improved; for example, self-attention or a combination of two attention 254 mechanisms could be used to obtain a better context vector. It is also possible to 255 use a combination of $\mathrm{CNN}$ and attention mechanism to obtain an excellent method 256 (Su et al. 2014). These possibilities warrant investigation. 


\section{Acknowledgments}

258 This work was supported by the Natural Science Foundation of China (No.

259 61902259), the Natural Science Foundation of Guangdong province (grant no.

260 2018A0303130084).

261 Declarations

262 Conflict of Interest sections

263

264

There is no conflict of interest in this paper and the data is available, which 265 can be obtained from https://github.com/huangqianfei0916/6ma-rice.

266 Author Contributions

Qianfei Huang and Wenyang Zhou are mainly responsible for the 268 development of experimental models and other work. Fei Guo, Lei Xu and Lichao 269 Zhang's main task was to guide the experiment.

270

271 


\section{References}

273 Basith S, Manavalan B, Shin TH, and Lee G. 2019. SDM6A: A web-based integrative machine-learning framework 274 for predicting 6mA sites in the rice genome. Molecular Therapy-Nucleic Acids 18:131-141.

275 Chen J, Han G, Xu A, and Cai H. 2019a. Identification of multidimensional regulatory modules through multi-graph matching with network constraints. IEEE Transactions on Biomedical Engineering 67:987-998.

277 Chen J, Peng H, Han G, Cai H, and Cai J. 2019b. HOGMMNC: a higher order graph matching with multiple 278 network constraints model for gene-drug regulatory modules identification. Bioinformatics 35:602-610.

279 Chen W, Lv H, Nie F, and Lin H. 2019c. i6mA-Pred: Identifying DNA N6-methyladenine sites in the rice genome. 280 Bioinformatics 35:2796-2800.

281

282

283

284 285

286

287

288

289

290

291

292

293

294

295

296

297 298

Cheng L. 2019a. Computational and Biological Methods for Gene Therapy. Curr Gene Ther 19:210. $10.2174 / 156652321904191022113307$

Cheng L. 2019b. Computational and Biological Methods for Gene Therapy. Current Gene Therapy 19:210-210.

Cheng L, and Hu Y. 2018. Human Disease System Biology. Curr Gene Ther 18:255-256. $10.2174 / 1566523218666181010101114$

Cheng L, Hu Y, Sun J, Zhou M, and Jiang Q. 2018a. DincRNA: a comprehensive web-based bioinformatics toolkit for exploring disease associations and ncRNA function. Bioinformatics 34:1953-1956. 10.1093/bioinformatics/bty002

Cheng L, Zhao H, Wang P, Zhou W, Luo M, Li T, Han J, Liu S, and Jiang Q. 2019. Computational Methods for Identifying Similar Diseases. Molecular therapy Nucleic acids 18:590-604.

Cheng L, Zhuang H, Yang S, Jiang H, Wang S, and Zhang J. 2018b. Exposing the Causal Effect of C-Reactive Protein on the Risk of Type 2 Diabetes Mellitus: A Mendelian Randomization Study. Front Genet 9:657. 10.3389/fgene. 2018.00657

Chu Y, Kaushik AC, Wang X, Wang W, Zhang Y, Shan X, Salahub DR, Xiong Y, and Wei DQ. 2019. DTI-CDF: a cascade deep forest model towards the prediction of drug-target interactions based on hybrid features. Briefings in Bioinformatics. 10.1093/bib/bbz152

Deng Y, Xu X, Qiu Y, Xia J, Zhang W, and Liu S. 2020. A multimodal deep learning framework for predicting drug-drug interaction events. Bioinformatics. 10.1093/bioinformatics/btaa501 
299

300

301

302

303

304

305

306

307

308

309

310

311

312

313

314

315

316

317

318

319

320

321

322

323

324

325

326

327

Ding Y, Tang J, and Guo F. 2019a. Identification of drug-side effect association via multiple information integration with centered kernel alignment. Neurocomputing 325:211-224. https://doi.org/10.1016/j.neucom.2018.10.028

Ding Y, Tang J, and Guo F. 2019b. Identification of Drug-Side Effect Association via Semisupervised Model and Multiple Kernel Learning. Ieee Journal of Biomedical and Health Informatics 23:2619-2632. 10.1109/jbhi.2018.2883834

Dou LJ, Li XL, Ding H, Xu L, and Xiang HK. 2020. Is There Any Sequence Feature in the RNA Pseudouridine Modification Prediction Problem? Molecular Therapy-Nucleic Acids 19:293-303. 10.1016/j.omtn.2019.11.014

Fang T, Zhang Z, Sun R, Zhu L, He J, Huang B, Xiong Y, and Zhu X. 2019. RNAm5CPred: Prediction of RNA 5Methylcytosine Sites Based on Three Different Kinds of Nucleotide Composition. Mol Ther Nucleic Acids 18:739-747. 10.1016/j.omtn.2019.10.008

Feng P, Yang H, Ding H, Lin H, Chen W, and Chou K-C. 2019. iDNA6mA-PseKNC: Identifying DNA N6methyladenosine sites by incorporating nucleotide physicochemical properties into PseKNC. Genomics 111:96-102.

Gong Y, Niu Y, Zhang W, and Li X. 2019. A network embedding-based multiple information integration method for the MiRNA-disease association prediction. BMC Bioinformatics 20:468. 10.1186/s12859-019-3063-3

Hao L, Dao F-Y, Guan Z-X, Zhang D, Tan J-X, Zhang Y, Chen W, and Lin H. 2019. iDNA6mA-Rice: a computational tool for detecting N6-methyladenine sites in rice. Frontiers in genetics 10:793.

He J, Fang T, Zhang Z, Huang B, Zhu X, and Xiong Y. 2018a. PseUI: Pseudouridine sites identification based on RNA sequence information. BMC Bioinformatics 19:306. 10.1186/s12859-018-2321-0

He W, Jia C, and Zou Q. 2019. 4mCPred: Machine Learning Methods for DNA N4-methylcytosine sites Prediction. Bioinformatics 35:593-601. 10.1093/bioinformatics/bty668

He W, Wei L, and Zou Q. 2018b. Research Progress in Protein Post-Translational Modification Site Prediction. Briefings in Functional Genomics 18:220-229.

Hu B, Zheng L, Long C, Song M, Li T, Yang L, and Zuo Y. 2019. EmExplorer: a database for exploring time activation of gene expression in mammalian embryos. Open Biol 9:190054. 10.1098/rsob.190054

Huang GH, and Li JC. 2018. Feature Extractions for Computationally Predicting Protein Post-Translational Modifications. Current Bioinformatics 13:387-395. 10.2174/1574893612666170707094916 
328

Huang Q, Zhang Y, Peng H, Dan T, Weng W, and Cai H. 2020a. Deep Subspace Clustering to Achieve Jointly Latent Feature Extraction and Discriminative Learning. Neurocomputing.

Huang QF, Zhang J, Guo F, and Zou Q. 2020b. 6mA-RicePred: A method for identifying DNA N6-methyladenine sites in the rice genome based on feature fusion. Frontiers in Plant Science 11:4.

Jin Q, Meng Z, Pham TD, Chen Q, Wei L, and Su R. 2019. DUNet: A deformable network for retinal vessel segmentation. Knowledge-Based Systems 178:149-162.

Kong L, and Zhang L. 2019. i6mA-DNCP: computational identification of DNA N6-methyladenine sites in the rice genome using optimized dinucleotide-based features. Genes 10:828.

Li J, Pu Y, Tang J, Zou Q, and Guo F. 2020. DeepAVP: a dual-channel deep neural network for identifying variable-length antiviral peptides. Ieee Journal of Biomedical and Health Informatics:1 - 1. 10.1109/JBHI.2020.2977091

Liu B. 2019. BioSeq-Analysis: a platform for DNA, RNA, and protein sequence analysis based on machine learning approaches. Briefings in Bioinformatics 20:1280-1294.

Liu B, Gao X, and Zhang H. 2019a. BioSeq-Analysis2.0: an updated platform for analyzing DNA, RNA, and protein sequences at sequence level and residue level based on machine learning approaches. Nucleic Acids Research 47:e127.

Liu B, Li C, and Yan K. DeepSVM-fold: Protein fold recognition by combining Support Vector Machines and pairwise sequence similarity scores generated by deep learning networks. Briefings in Bioinformatics DOI: 10.1093/bib/bbz098.

Liu B, and Li K. 2019. iPromoter-2L2.0: identifying promoters and their types by combining Smoothing Cutting Window algorithm and sequence-based features. Molecular Therapy-Nucleic Acids 18:80-87.

Liu D, Li G, and Zuo Y. 2019b. Function determinants of TET proteins: the arrangements of sequence motifs with specific codes. Brief Bioinform 20:1826-1835. 10.1093/bib/bby053

Liu Z, Dong W, Jiang W, and He Z. 2019c. csDMA: an improved bioinformatics tool for identifying DNA 6 mA modifications via Chou's 5-step rule. Scientific reports 9:1-9.

Patil K, and Chouhan U. 2019. Relevance of Machine Learning Techniques and Various Protein Features in Protein Fold Classification: A Review. Current Bioinformatics 14:688-697. $10.2174 / 1574893614666190204154038$

Ren F, Yang C, Qiu Q, Zeng N, Cai C, Hou C, and Zou Q. 2019. Exploiting Discriminative Regions of Brain Slices 
357

358

359

360

361

362

363

364

365

366

367

368

369

370

371

372

373

374

375

376

377

378

379

380

381

382

383

384

385

based on 2D CNNs for Alzheimer's Disease Classification. Ieee Access 7:181423 - 181433.

Shan X, Wang X, Li CD, Chu Y, Zhang Y, Xiong Y, and Wei DQ. 2019. Prediction of CYP450 Enzyme-Substrate Selectivity Based on the Network-Based Label Space Division Method. Journal of Chemical Information and Modeling 59:4577-4586. 10.1021/acs.jcim.9b00749

Shen Y, Ding Y, Tang J, Zou Q, and Guo F. 2019a. Critical evaluation of web-based prediction tools for human protein subcellular localization. Briefings in Bioinformatics. 10.1093/bib/bbz106

Shen Y, Tang J, and Guo F. 2019b. Identification of protein subcellular localization via integrating evolutionary and physicochemical information into Chou's general PseAAC. Journal of Theoretical Biology 462:230-239. https://doi.org/10.1016/j.jtbi.2018.11.012

Su J, Wang Y, Xing X, Liu J, and Zhang Y. 2014. Genome-wide analysis of DNA methylation in bovine placentas. BMC Genomics 15:12. 10.1186/1471-2164-15-12

Su R, Liu X, Wei L, and Zou Q. 2019a. Deep-Resp-Forest: A deep forest model to predict anti-cancer drug response. Methods 166:91-102. 10.1016/j.ymeth.2019.02.009

Su R, Wu H, Xu B, Liu X, and Wei L. 2019b. Developing a Multi-Dose Computational Model for Drug-induced Hepatotoxicity Prediction based on Toxicogenomics Data. IEEE/ACM Transactions on Computational Biology and Bioinformatics 16:1231-1239.

Sun J, Zhang Z, Bao S, Yan C, Hou P, Wu N, Su J, Xu L, and Zhou M. 2020. Identification of tumor immune infiltration-associated lncRNAs for improving prognosis and immunotherapy response of patients with non-small cell lung cancer. J Immunother Cancer 8. 10.1136/jitc-2019-000110

Wang H, Ding Y, Tang J, and Guo F. 2020a. Identification of membrane protein types via multivariate information fusion with Hilbert-Schmidt Independence Criterion. Neurocomputing 383:257-269. 10.1016/j.neucom.2019.11.103

Wang Z, He W, Tang J, and Guo F. 2020b. Identification of Highest-Affinity Binding Sites of Yeast Transcription Factor Families. Journal of Chemical Information and Modeling 60:1876-1883. 10.1021/acs.jcim.9b01012

Wei L, Hu J, Li F, Song J, Su R, and Zou Q. 2018a. Comparative analysis and prediction of quorum-sensing peptides using feature representation learning and machine learning algorithms. Briefings in Bioinformatics. 10.1093/bib/bby 107

Wei L, Zhou C, Chen H, Song J, and Su R. 2018b. ACPred-FL: a sequence-based predictor based on effective feature representation to improve the prediction of anti-cancer peptides. Bioinformatics 34:4007-4016. 
386

387

388

389

390

391

392

393

394

395

396

397

398

399

400

401

402

403

404

405

406

407

408

409

410

411

412

413

414

Xia W, Xu J, Yu G, Yao G, Xu K, Ma X, Zhang N, Liu B, Li T, Lin Z, Chen X, Li L, Wang Q, Shi D, Shi S, Zhang Y, Song W, Jin H, Hu L, Bu Z, Wang Y, Na J, Xie W, and Sun YP. 2019. Resetting histone modifications during human parental-to-zygotic transition. Science 365:353-360. 10.1126/science.aaw5118

Xiong Y, Wang Q, Yang J, Zhu X, and Wei DQ. 2018. PredT4SE-Stack: Prediction of Bacterial Type IV Secreted Effectors From Protein Sequences Using a Stacked Ensemble Method. Front Microbiol 9:2571. 10.3389/fmicb.2018.02571

Xu A, Chen J, Peng H, Han G, and Cai H. 2019a. Simultaneous interrogation of cancer omics to identify subtypes with significant clinical differences. Frontiers in Genetics 10:236.

Xu B, Liu D, Wang Z, Tian R, and Zuo Y. 2020a. Multi-substrate selectivity based on key loops and nonhomologous domains: new insight into ALKBH family. Cell Mol Life Sci. 10.1007/s00018-020-03594-9

Xu H, Hu R, Jia P, and Zhao Z. 2020b. 6mA-Finder: a novel online tool for predicting DNA N6-methyladenine sites in genomes. Bioinformatics 36:3257-3259.

Xu L, Liang G, Liao C, Chen G-D, and Chang C-C. 2018a. An Efficient Classifier for Alzheimer's Disease Genes Identification. Molecules 23:3140.

Xu L, Liang G, Liao C, Chen G-D, and Chang C-C. 2019b. k-Skip-n-Gram-RF: A Random Forest Based Method for Alzheimer's Disease Protein Identification. Frontiers in Genetics 10. 10.3389/fgene.2019.00033

Xu L, Liang G, Shi S, and Liao C. 2018b. SeqSVM: A Sequence-Based Support Vector Machine Method for Identifying Antioxidant Proteins. International Journal of Molecular Sciences 19:1773.

Xu L, Liang G, Wang L, and Liao C. 2018c. A Novel Hybrid Sequence-Based Model for Identifying Anticancer Peptides. Genes 9:158.

Yan C, Zhang Z, Bao S, Hou P, Zhou M, Xu C, and Sun J. 2020. Computational Methods and Applications for Identifying Disease-Associated lncRNAs as Potential Biomarkers and Therapeutic Targets. Mol Ther Nucleic Acids 21:156-171. 10.1016/j.omtn.2020.05.018

Yu H, and Dai Z. 2019. SNNRice6mA: a deep learning method for predicting DNA N6-methyladenine sites in rice genome. Frontiers in genetics 10:1071.

Zhang W, Jing K, Huang F, Chen Y, Li B, Li J, and Gong J. 2019a. SFLLN: A sparse feature learning ensemble method with linear neighborhood regularization for predicting drug-drug interactions. Information Sciences 497:189-201. https://doi.org/10.1016/j.ins.2019.05.017

Zhang W, Li Z, Guo W, Yang W, and Huang F. 2019b. A fast linear neighborhood similarity-based network link 
415

416

417

418

419

420

421

422

423

424

425

426

427

428

429

430

431

432

433

inference method to predict microRNA-disease associations. IEEE/ACM Trans Comput Biol Bioinform. 10.1109/TCBB.2019.2931546

Zhou M, Hu L, Zhang Z, Wu N, Sun J, and Su J. 2018. Recurrence-Associated Long Non-coding RNA Signature for Determining the Risk of Recurrence in Patients with Colon Cancer. Mol Ther Nucleic Acids 12:518-529. 10.1016/j.omtn.2018.06.007

Zhou M, Zhao H, Xu W, Bao S, Cheng L, and Sun J. 2017. Discovery and validation of immune-associated long non-coding RNA biomarkers associated with clinically molecular subtype and prognosis in diffuse large B cell lymphoma. Mol Cancer 16:16. 10.1186/s12943-017-0580-4

Zhu X, He J, Zhao S, Tao W, Xiong Y, and Bi S. 2019. A comprehensive comparison and analysis of computational predictors for RNA N6-methyladenosine sites of Saccharomyces cerevisiae. Brief Funct Genomics 18:367376. $10.1093 / \mathrm{bfgp} / \mathrm{elz} 018$

Zou Q. 2019. Latest Machine Learning Techniques for Biomedicine and Bioinformatics. Current Bioinformatics 14:176-177. 10.2174/157489361403190220112855

Zou Q, and Ma Q. 2019. The application of machine learning to disease diagnosis and treatment. Mathematical Biosciences 320:108305. 10.1016/j.mbs.2019.108305

Zou Q, Xing P, Wei L, and Liu B. 2019. Gene2vec: Gene Subsequence Embedding for Prediction of Mammalian N6-Methyladenosine Sites from mRNA. rna 25:205-218. 10.1261/rna.069112.118

Zuo Y, Li Y, Chen Y, Li G, Yan Z, and Yang L. 2017. PseKRAAC: a flexible web server for generating pseudo Ktuple reduced amino acids composition. Bioinformatics 33:122-124. 10.1093/bioinformatics/btw564 
Table $\mathbf{1}$ (on next page)

Table 1 | All datasets. 


\begin{tabular}{cccc}
\hline Dataset & Positive & Negative & Total \\
\hline Rice & 154000 & 154000 & 308000 \\
Mus musculus & 1934 & 1934 & 3868 \\
Human_Train & 491885 & 491885 & 983770 \\
Human_Test & 122971 & 122971 & 245942 \\
\hline
\end{tabular}

1 
Table 2 (on next page)

Table 2 | The parameters of each experiment. 


\begin{tabular}{lllll}
\hline Experiment & Ir & hidden_dim & dropout & Bach_size \\
\hline Fig 2 & 0.001 & 100 & 0.3 & 64 \\
Table 3 & 0.001 & 100 & 0.3 & 64 \\
Table 4-cv & 0.005 & 100 & 0.3 & 64 \\
Table 4-ind & 0.005 & 100 & 0.3 & 64 \\
Table 5 & 0.001 & 100 & 0.3 & 64 \\
\hline
\end{tabular}




\section{Table 3 (on next page)}

Table 3 | Performance comparison between $6 \mathrm{~mA}$-Pred and other methods via 5-fold cross validation based on the rice dataset. 


\begin{tabular}{lccccc}
\hline Method & Sn $(\%)$ & Sp $(\%)$ & Acc $(\%)$ & MCC & AUC \\
\hline SNNRice6mA & 93.67 & 86.74 & 90.20 & 0.81 & 0.96 \\
SNNRice6mA- & 94.33 & 89.75 & 92.04 & 0.84 & 0.97 \\
large & & & & & \\
iDNA6mA- & 93.00 & 90.50 & 91.70 & 0.84 & 0.96 \\
rice & & & & & \\
6mA-Pred & 95.66 & 92.38 & 94.02 & 0.88 & 0.981 \\
\hline
\end{tabular}




\section{Table 4(on next page)}

Table 4 | Performance of $6 \mathrm{~mA}$-Pred evaluated via 5 -fold cross validation and independent testing based on the Mus musculus dataset. 


\begin{tabular}{lccccc}
\hline Method & Sn (\%) & Sp (\%) & Acc (\%) & MCC & AUC \\
\hline 6mA-Pred-cv & 93.8 & 98.5 & 96.1 & 0.92 & 0.981 \\
6mA-Pred- & 87.8 & 98.4 & 93.8 & 0.861 & 0.949 \\
ind & & & & & \\
IDNA6mA- & 93.28 & 100 & 96.73 & 0.93 & - \\
PseKNC & & & & \\
\hline
\end{tabular}




\section{Table 5 (on next page)}

Table 5 | Performance of $6 \mathrm{~mA}$-Pred evaluated via independent testing based on the human dataset. 


\begin{tabular}{lccccc}
\hline Method & Sn (\%) & Sp (\%) & Acc (\%) & MCC & AUC \\
\hline 6mA-Pred- & 93.28 & 94.2 & 93.34 & 0.87 & 0.98 \\
ind & & & & \\
SICD6mA & 93.33 & 95.00 & 93.66 & 0.874 & - \\
\hline
\end{tabular}


Figure 1

Figure 1 | A flow chart of the structure of $6 \mathrm{~mA}$-Pred.

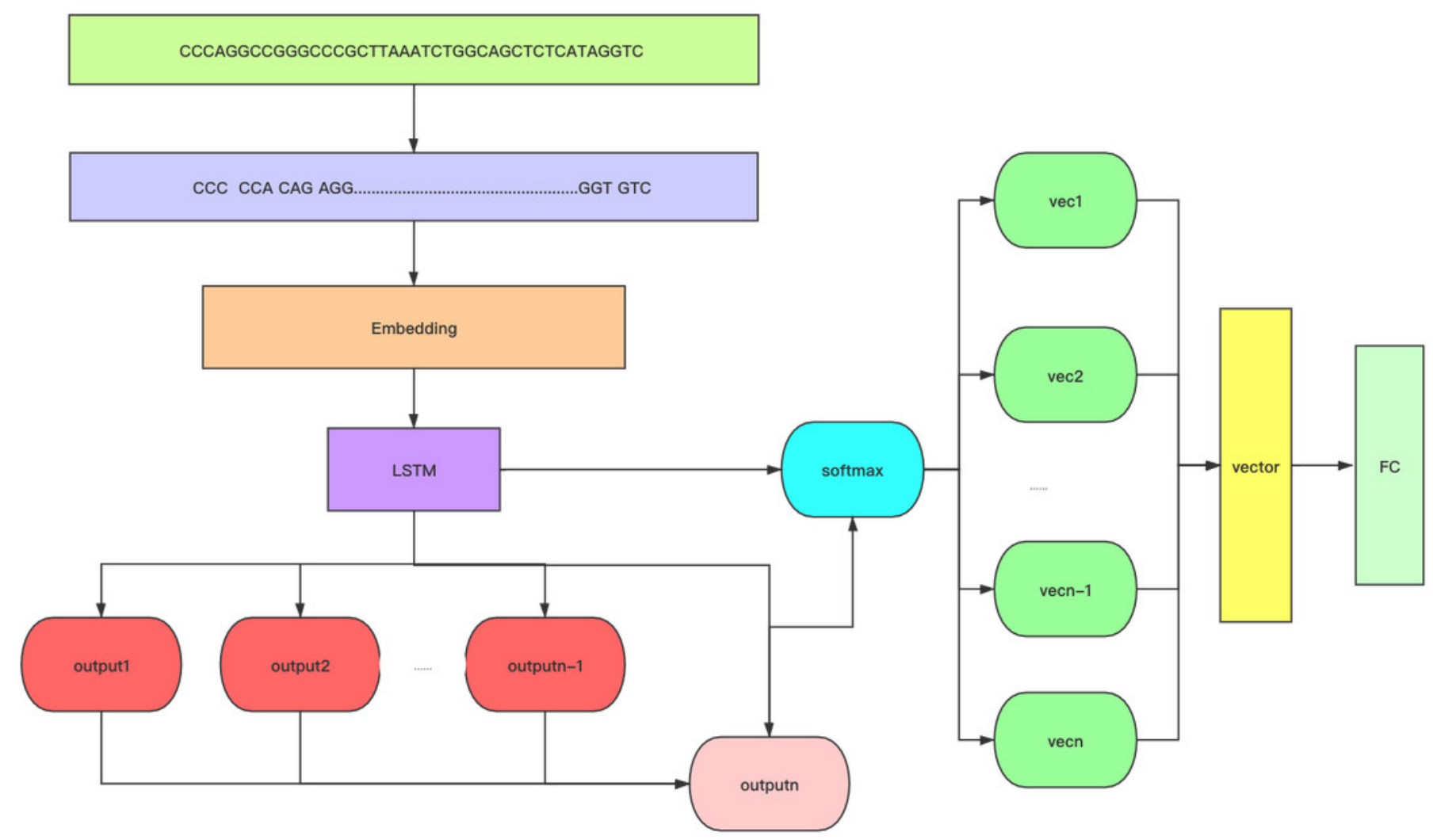


Figure 2

Figure 2 | Performance of $6 \mathrm{~mA}$-Pred evaluated via independent testing based on different k-values.

A

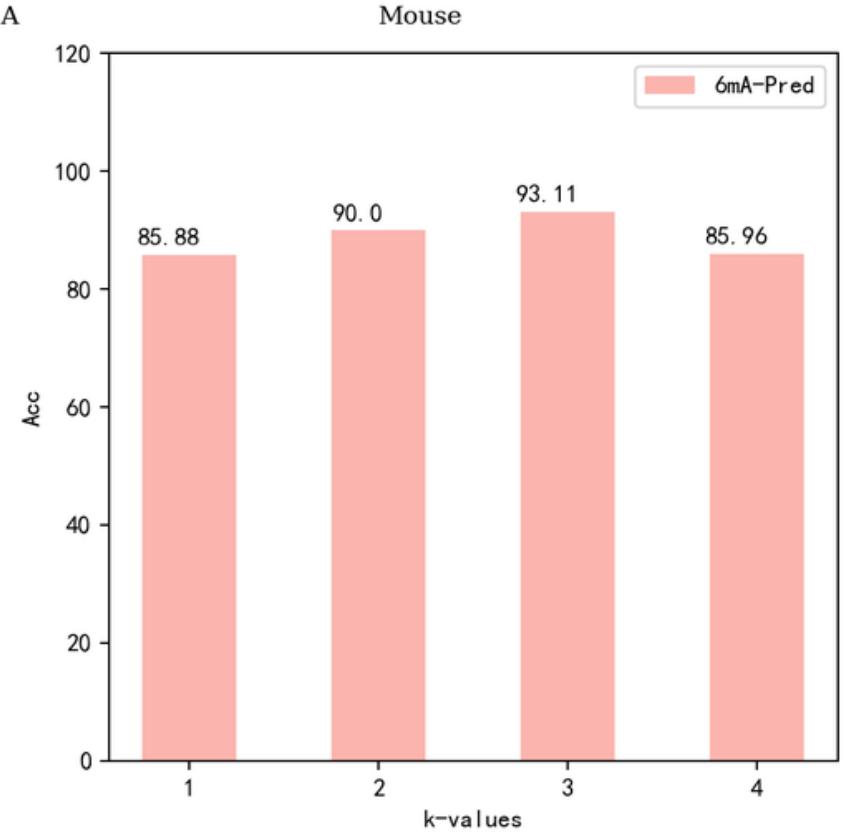

B

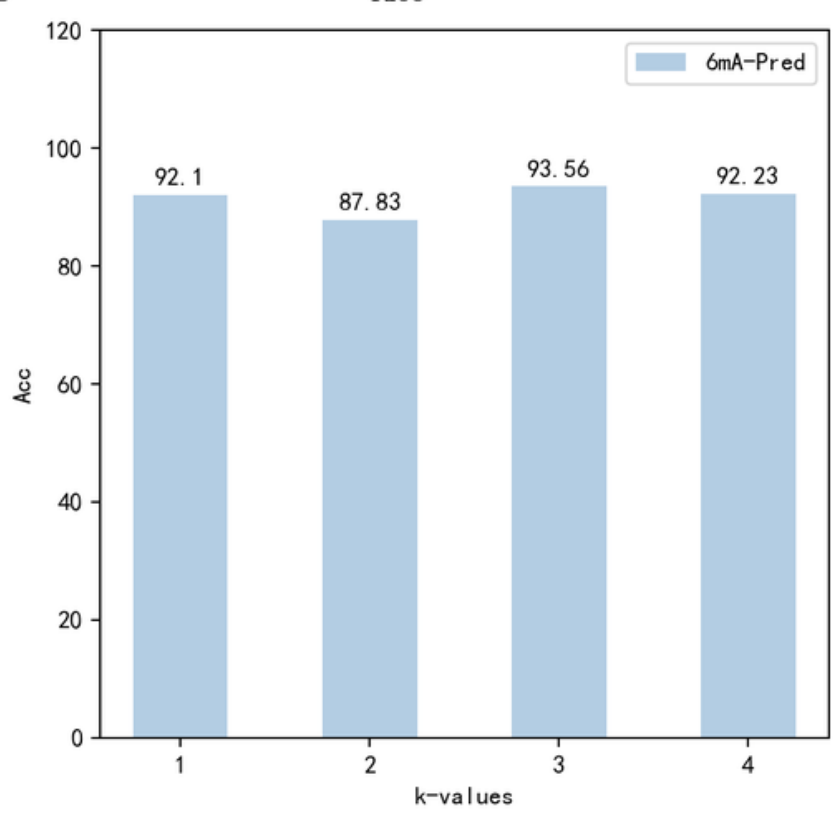

C

Human

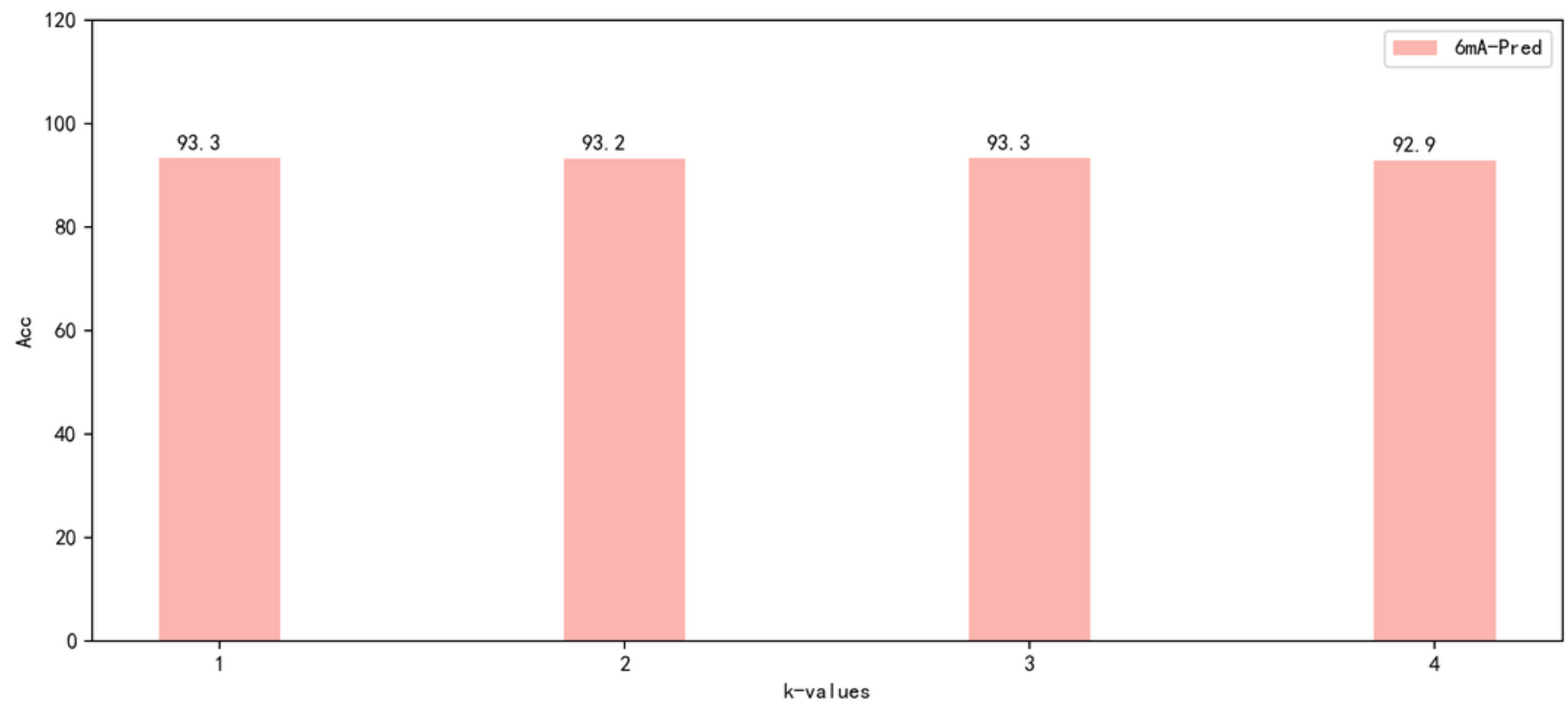


Figure 3

Figure 3 | Predictive performance at different ratios for the rice dataset.
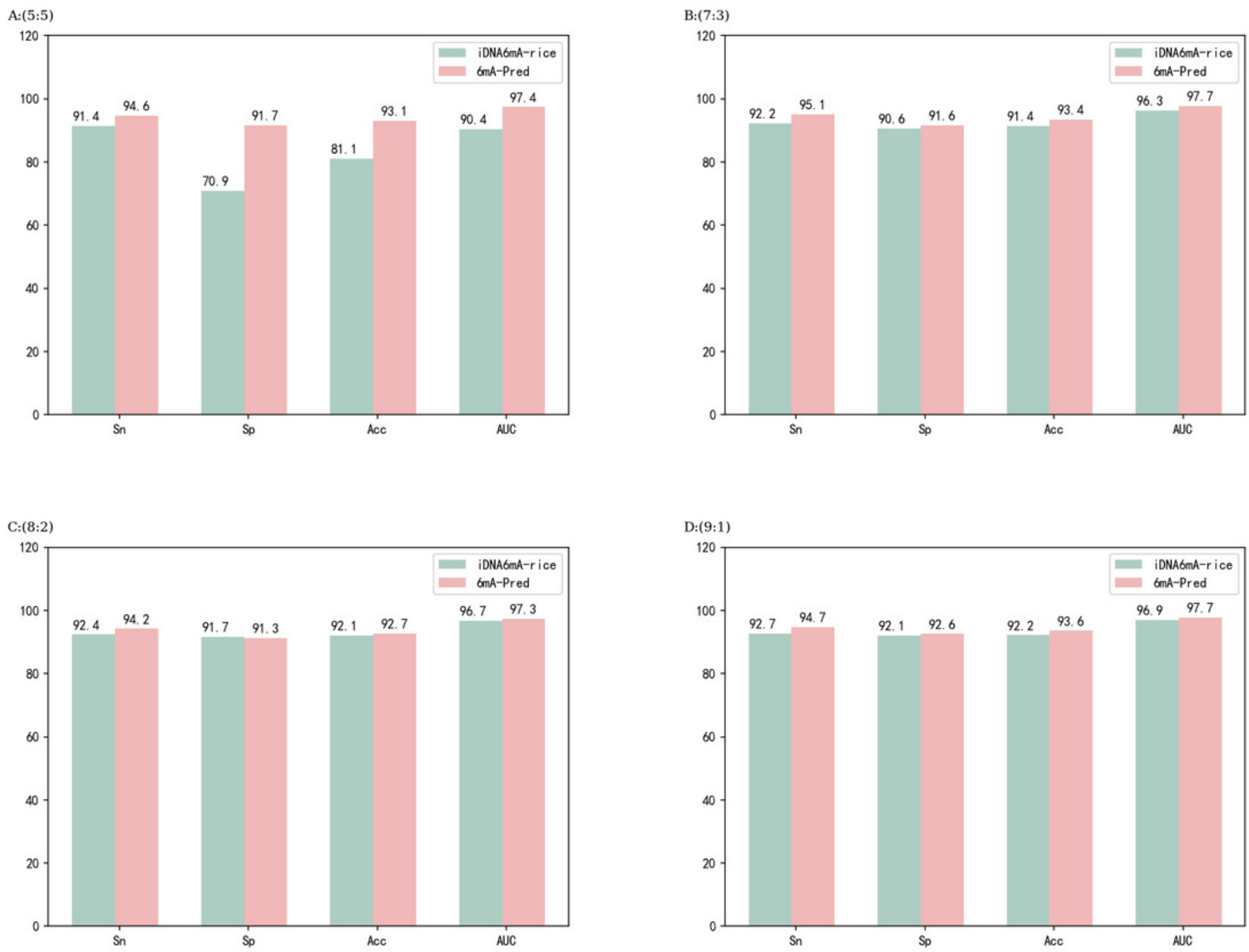
Figure 4

Figure $4 \square$ Performance comparison between $6 \mathrm{~mA}$-Pred and other machine learning methods independent testing based on all datasets.
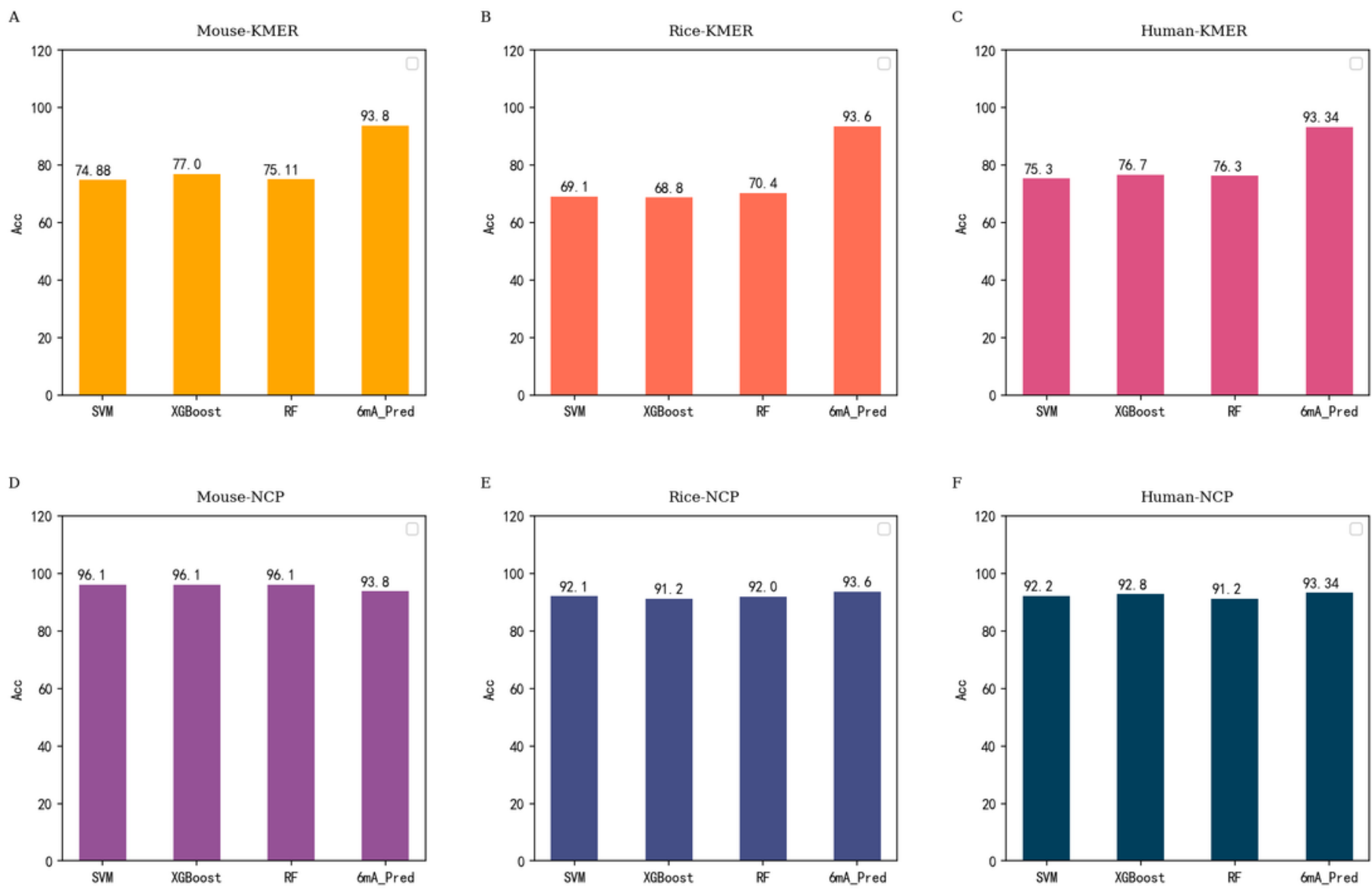